\title{
Computer vision System for Public Illumination Management
}

\author{
Peterson Belan \\ Informatics and Knowledge \\ Management Graduate Program, \\ Nove de Julho University, \\ São Paulo, Brazil
}

\author{
Anderson S. Vanin \\ Informatics and Knowledge \\ Management Graduate Program, \\ Nove de Julho University, \\ São Paulo, Brazil
}

\author{
Edward Netzer \\ Informatics and Knowledge \\ Management Graduate Program, \\ Nove de Julho University, \\ São Paulo, Brazil
}

\begin{abstract}
The present work proposes the management of street lighting through a computer vision approach, for which algorithms are used to detect pedestrians. The current scenario of demand for electricity, has rates that constantly increase, due to taxes, urban expansion, among others. Therefore, it is extremely important to look for alternative ways to minimize costs. One of the segments to be explored with great economic potential is the management of street lighting, in recent times changes have been taking place in this area, where governments are replacing sodium vapor lighting by LEDs lamps, which are already capable of dramatically reduce energy consumption. In this context, computer vision systems can help to reduce this consumption even further, controlling the power of these LED lamps according to the flow of people on the road. The computer vision system proposed in this work was implemented in $\mathrm{C}++$ using the OpenCV library, applied in a Raspberry Pi 3. It was also used the Fuzzy Logic to calculate the power that the LEDs must be adjusted due to the number of people on the road as well as the ambient lighting. For the execution of the validation tests of this proposal, images were acquired on public roads with pedestrians, as well as simulations of these environments were carried out, thus being possible to test all the proposed possibilities. With the real application of this project, it is possible to observe a savings of approximately $44 \%$ in the consumption of public lighting, this compared to the use of LED lighting.
\end{abstract}

\section{Keywords}

Computer Vision, Pedestrian Detection, Street Lighting, Fuzzy Logic, Smart Cities

\section{INTRODUCTION}

The current scenario of electricity demand has tariffs that increase constantly, due to taxes, urban expansion, among others. Therefore, it is of paramount importance to seek alternative means to minimize costs. One of the segments to be explored with great economic potential is public road lighting management [1]. Another important point nowadays is the use of technological resources to improve the planning and development of cities in an organized and intelligent way, thus favoring the growth of large metropolis [2].

Public lighting has evolved over time in the mid19th century, where since the 19th century, moving from gas and oil lamps, then moving to the use of electricity with incandescent bulbs, which are currently being replaced by LED (Light Emitting Diode) [3].

The lighting of public roads is essential in cities, roads and sidewalks, as it provides citizens with safety and comfort in their journey [4]. As an example of the cost of public lighting in the state of São Paulo in Brazil, the cost of public lighting corresponded to $2.38 \%$ and all electricity consumption in the city, equivalent to $823,899.76 \mathrm{MWh}$ in 2017 [5]. A possible approach to cost reduction is the use of intelligent techniques and computer vision.

Computer vision is a set of computational techniques that aims to estimate or explain the geometric and dynamic properties of a given object from digital images [6]. Several applications are found in the literature in the recognition of people, which employ different techniques $[7,8]$.

Integrating facilities and equipment to people's affordable and efficient use, basically forms the concept of "Smart Cities", bringing a wide range of implementations such as: public safety, energy saving, road pedestrian detection (sidewalks), automated cities with learning algorithms, among others, in order to significantly improve the management of a city's energy resources. The system of control and management of public lighting is an integral part of this concept as an effective method of managing energy resources [9].

This work tries to contribute to the development of an efficient methodology with the use of computer vision and Fuzzy Logic [10] to control a hardware that manages public LED street lighting and a movement of pedestrians on roads, using electricity savings.

\subsection{Street lighting management}

The waste with electricity in public lighting has enormous economic and environmental consequences, making an electric energy management system increasingly necessary in the current economic scenario of cities, helping in the control and power down of lighting when necessary. It is possible to save considerable amounts of energy by controlling lighting based on pedestrian presence [11].

The luminaire model with LED lamp currently used and on public roads are around $280 \mathrm{~W}$ and generate a luminous flow of $28000 \mathrm{~lm}$. Almost control is currently per season hour of the year, where luminaires are lit from approximately $7 \mathrm{pm}$ to $7 \mathrm{am}$, around 12 hours connected with full load. The consumption calculation is given by Equation 1 .

$$
\text { Consumption }=\mathrm{T} * \mathrm{P}
$$

Therefore, considering the time $(\mathrm{T})$ in hours and power $(\mathrm{P})$ in Watts, a luminaire consumes around $3,36 \mathrm{~kW} / \mathrm{h}$ or $100,8 \mathrm{~kW}$ month. According to ENEL 2019 [12] the value of $\mathrm{kWh}$ for public lighting in Brazil is US\$ 0.12, generating monthly expense per unit of approximately US\$ 5.63. 


\section{RELATED WORKS}

The study addresses a system for integrating static converters applied to public lighting systems [13]. At first the author proposes that magnetic reactors be switched to electronic reactors and added levels of light intensity for each period of the night (dimerize the lamps according to the time). The second stage of the study, an acoustic resonance analysis was performed at high frequency, then choosing a square wave feed to avoid resonance. From this it was noted that it would be necessary to use a current limiting equipment. With electronic reactors it is possible to ensure stable control in the power factor. Switching High-Pressure Mercury (HPM) lamps to HPS (High-Pressure Sodium). And with these changes, the energy economy spent has reached considerable savings.

According to [14] the electrothermal design methodology of LEDs applied to the development of public lighting systems aims to provide indicative of the best point of operation of the LED, an analysis of the life of the LEDs leading in consideration of thermal, electrical and photometric characteristics, and to avoid heating several tests with various models and sizes of heatsink. And so, proposed a static electrothermal model, using to develop an electrothermal methodology that can be applied to any lighting system with LEDs working on a permanent basis. A control system was also developed for LEDs drive, which could make temperature and current control composed of Buck-Boost converters (it is an electronic circuit capable of converting a cc voltage into another cc polarity voltage the initial goal of being comparatively better than the most widely used public lighting system so far is thus increasing the life of the LEDs.

The research of [15] presents a system of street lighting, using LED for lighting, powered by batteries that in turn are charged by solar panels. The idea of implementing this project for public lighting was focused on areas restricted to the supply of electricity such as farms and unpaved roads. The author did several laboratory tests to identify the efficiency of the prototype, and it was concluded that through the behavior of the battery charge current and the current panels results in a low variation, so there was a better use of the solar panel and also in the conversion of life.

According to [4] conventional public lighting systems, they do not have an efficient system of optimal electricity management, generating unnecessary expenses with lights on throughout the night, thus justifying the emergence of technologies lighting management. The proposed system is based on the location of pedestrians through the frequency emitted by each pedestrian's mobile device to increase or reduce the illuminated area around passers-by, where each lamp pole is has a ZigBee radio device. Some problems were encountered regarding signal reception from cellular devices and that for correction and robustness GPRS antennas would be required at the lampposts.

The article proposed by [16] explores human recognition by surveillance cameras for the way the person walks. The authors discuss imaging segmentation techniques through binary images and their conversion into vectors, so the difference between the vectors generated indicates an individualized walking style. All calculation is done using the euclidean distance of two vectors. Although the results presented are very promising, the system still needs more experiments when compared to traditional models.

In [17] was proposed an experiment with three thermal cameras, monitoring the intensity of people's movement and trajectories to be used as variables to control lighting. The authors also evaluate the effects caused by the change in light intensity on pedestrians in relation to their behavior and highlight that pedestrians outside the controlled area could notice the changes, but to those who were inside these limits were not significantly noted. There was a reduction of around $90 \%$ in electricity consumption.

The authors of [18] proposes a real-time adaptive approach in the lighting scheme, which detects the presence of vehicles and pedestrians, and dynamically adjusts brightness to the ideal level by improving energy efficiency in street lighting using propagation communication signal between nodes via wireless network.

[11] address the use of human recognition using the computer vision technique for the management of electricity applied in public lighting of a school campus. The OpenCV library was used together with the Raspberry Pi. Although the project meets the initial objective, some gaps in the research were identified because it is a small area requiring further studies for large-scale employment.

\section{PROPOSED SYSTEM}

This project aims to bring an option of public lighting control in an intelligent and selective way, differentiating one or more pedestrians from a vehicle or animal, taking into account the ambient light in which the scene is found at the time of detection, so that can provide lighting in a balanced way. Consequently, to reduce unnecessary energy use and thus bring more energy savings and longer lamp life. It was considered that the minimum lighting of the pole, during the night period with no person detected, has a value of approximately $30 \%$ of the total power.

For this, an algorithm was developed in $\mathrm{C}++$ using the OpenCV ${ }^{1}$ library. The first step consists of the acquisition of the image by a webcam, then initializes the parameters for the image descriptor HOG, which is used to extract characteristics in ways that resemble a person in the image, thus sending his histogram vector to an SVM classifier trained to perform this type of classification, thus demarcating the image with rectangles at the place where the algorithm detected the characteristics of one or more people. Then the algorithm separates the image into quadrants, in this case three horizontal regions to obtain depth and distance information that the person is from the camera.

After the detection of pedestrians, there is the lighting control through fuzzy logic, which leads uses as input parameters the amount of people detected and the ambient lighting monitored by a luxmeter. Then data processing occurs based on input values thus generating its output. The value obtained by Fuzzy Inference System is used as an input parameter in a dimmer coupled to the equipment itself that processing the images.

\subsection{Proposed fuzzy system}

Fuzzy logic graphs were generated in the MATLAB ${ }^{2}$ computing environment using the Fuzzy Toolbox. Based on [19], seven linguistic variables were used, as they present an increase in accuracy by about $15 \%$ in relation to lower values. Three tests were performed empirically to establish fuzzy rules.

Seven triangular pertinence functions were assigned for the variables of the first entry, which is the existing lighting level in the environment, given in percentage: VeryLow, Low,

OpenCV - https://opencv.org/

Matlab - https://www.mathworks.com/products/matlab.html 
LittleLow, Normal, LittleHigh, High, VeryHigh. The second entry having 6 triangular pertinences function, being the number of people present: VeryFew, Few, Some, Group, Enough, Many. The output variable was the dimerization of light, in percentage, also with seven triangular pertinence functions being them: MinPower, LowPower, SmallPower, MedianPower, StrongPower, HighPower, MaxPower. The arrangement of the sets for inputs and outputs are displayed in Fig 1,2 , and 3 .

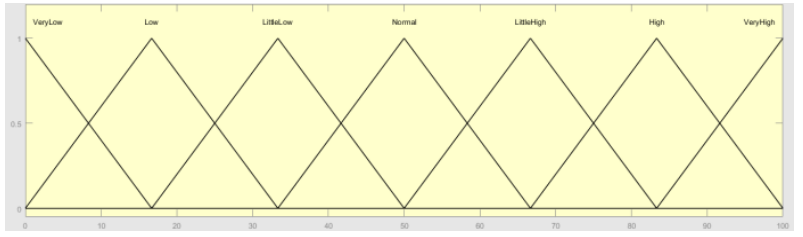

Figure 1. Fuzzy rule for ambient luminosity in \%

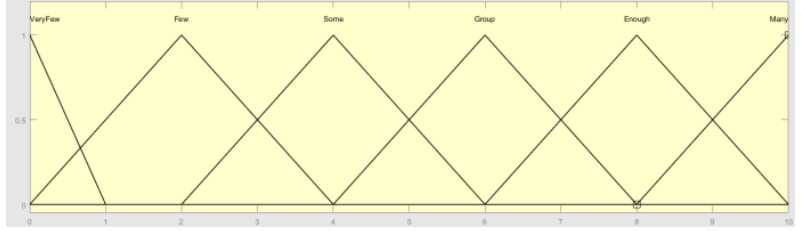

Figure 2. Fuzzy rule for number of people

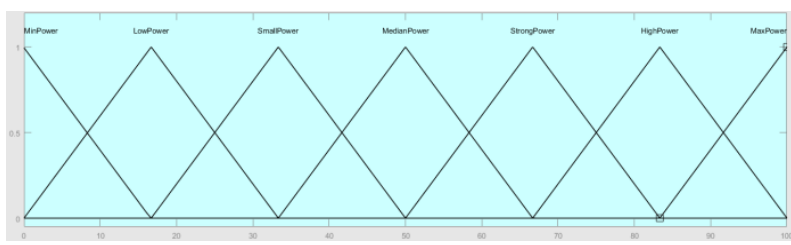

Figure 3. Fuzzy output for dimerization of the lighting

Table 1 displays the fuzzy inference rules using TakagiSugeno model. The number of rules, which represent the output, is the product of the number of sets of each input variable, totaling 42 ( 7 sets of ambient lighting x 6 sets of the number of people present).

Table 1. Fuzzy inference rules

\begin{tabular}{c|c|c|c|c|c|c|c}
\hline Ambient Light/People & VeryLow & Low & LittleLow & Normal & LittleHigh & High & VeryHigh \\
\hline VeryFew & SmallPower & SmallPower & SmallPower & SmallPower & MinPower & MinPower & MinPower \\
Some & LowPower & LowPower & LowPower & LowPower & SmallPower & SmallPower & MinPower \\
Group & MedianPower & MedianPower & MedianPower & LowPower & SmallPower & SmallPower & MinPower \\
Enough & StrongPower & StrongPower & MedianPower & MedianPower & LowPower & SmallPower & MinPower \\
Many & HighPower & HighPower & StrongPower & MedianPower & LowPower & LowPower \\
MaxPower & HighPower & StrongPower & StrongPower & LowPower & LowPower \\
\hline
\end{tabular}

\subsection{Algorithm for people detection}

For development of the proposed algorithm, the Code::Blocks3 IDE and OpenCV library were employed. The $\mathrm{C} / \mathrm{C}++$ language was used for building the application and the flowchart present in Fig 4 describes the whole process. As image descriptor for people detection was used HOG and SVM as classifier. The camera used in the experiments was a Logitech C920.

The first step of the algorithm is load HOG descriptors parameters and SVM classifier. The HOG descriptor has been widely used successfully in the task of detecting pedestrians and is present in the OpenCV library [20,21]. Briefly, this method describes an image from the value of the distribution of the directions (histograms) of the oriented gradients. These gradients have higher values in the regions of the image that have abrupt variations in intensity, that is, at the edges of objects and corners of the shapes, which contrast with the background [21].

The support vector machine, SVM, is a supervised machine learning algorithm that performs classification [22]. A supervised and linear learning algorithm is based on the use of two predefined classes from a database, to then designate which of these classes new input data fall into [23]. When applied to a computer vision process, SVM uses as input the characteristics already filtered from the image, whether these borders, colors or formats.

In the next step the image is acquired at resolution $1920 \times 1080$ pixels. Once the image is acquired, it can be processed by HOG and SVM, with its output is possible to get the number of people in the image. In order to optimize lighting control, the image was divided into three equal zones, and the number of people are grouped by these zones. These zones were created considering that one camera can control three lampposts.

The next step of the process is to read the ambient illumination, this is one input parameter for Fuzzy logic control. As input for the Fuzzy logic, the data of the three zones are computed separately, so the algorithm generate three outputs that are applied in the illumination control driver for dimerize the LEDs.

To conduct the experiments, a set of 150 images were acquired in a real environment (a public way), and in a controlled environment (lightning), to simulate all conditions proposed in this work. These images were divided in two groups, the first one, with 50 images, were used for parameterization of HOG descriptors and SVM classifier. The other 100 images were used for the proposed algorithm validation. In addition, more 100 images were acquired in a simulated environment, these images have been used only in validation process.

${ }^{3}$ Code:: Blocks IDE - http://www.codeblocks.org/ 


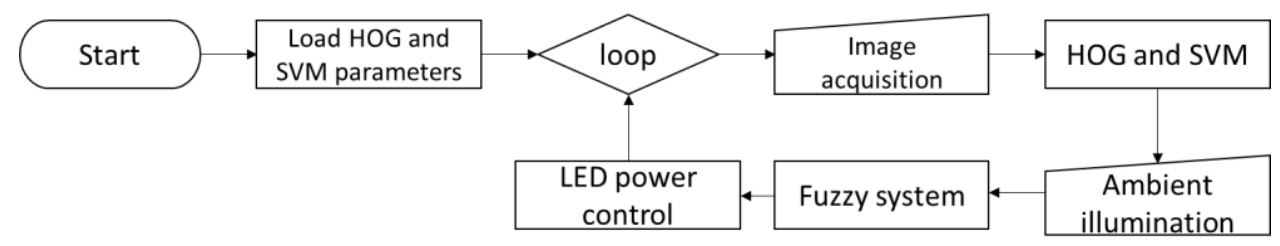

Figure 4. Steps of the proposed algorithm for people detection

\section{RESULTS AND DISCUSSION}

The people detection experiments were carried out in a real environment where it was noted that distance is a highly influential factor in detection. This influence was crucial for determine the angle and the area that is possible to recognize the people on the image. Based on this factor, it was determined that one camera can acquire images from three lampposts. During initial experiments, several false positives occurred, however after adjusting the appropriate conditions and the parameters of HOG and SVM this problem was circumvented. The average processing time for each image in the Raspberry Pi 3 was $4 \mathrm{~s}$.

After the adjustments made in the HOG and SVM, the hit rate in the real environment (Figure 5) was approximately $80 \%$, considering that these algorithms presented more false positives than false negatives. The 50 images of the public ways which was used to perform all adjustments of the algorithms were not used in evaluate the results, for that purpose another 100 images were used. These results can be considered adequate, considering the simplicity of the techniques used and enabling their use in low-cost equipment, such as the Raspberry Pi 3.

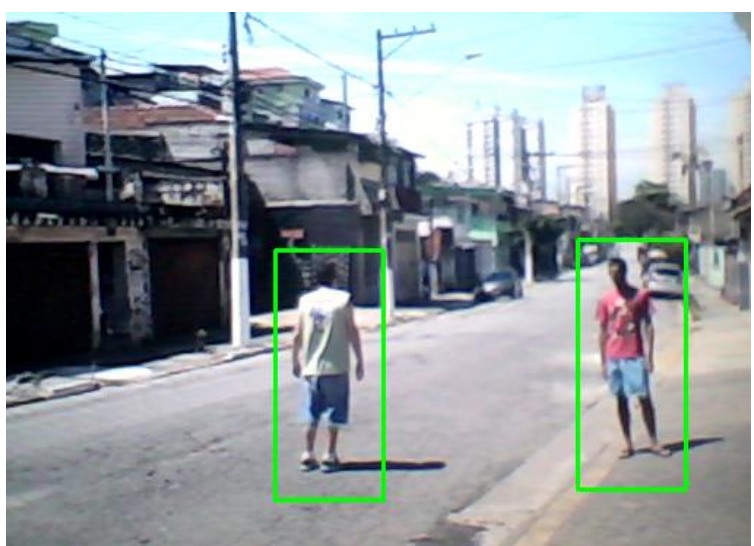

Figure 5. Example of an image from real environment

To validate the experiments related to lighting control (dimerization), experiments were carried out in a controlled environment, in this case a model was built to validate the problem. Considering this controlled environment, it was also possible to perform the variation of ambient light, simulating all possible conditions within the proposal of this work.

This experiment, 100 images were evaluated, which the worst results were presented with low natural lighting and in the condition with the minimum power of light supply, which can be explained by the webcam characteristics used in this work. For better results, an infrared camera must be used. The success rate in these images was $85 \%$, showing the feasibility of the proposal. Table 2 shows the results in detail obtained in real and controlled environment,
Table 2. Estimate and calculation of consumption by time

\begin{tabular}{c|c|c|c|c}
\hline Environment & Images & $\begin{array}{c}\text { Correct } \\
\text { detection (\%) }\end{array}$ & $\begin{array}{c}\text { False } \\
\text { positives (\%) }\end{array}$ & $\begin{array}{c}\text { False } \\
\text { negatives (\%) }\end{array}$ \\
\hline Real & 100 & 80 & 14 & 6 \\
\hline Controlled & 100 & 85 & 11 & 4 \\
\hline Final & 200 & 82.5 & 12.5 & 5 \\
\hline
\end{tabular}

However, it is clear the need for further investigation to improve the hit rate, whether with the application of new techniques or improvements in the hardware used.

Regarding the monitoring of ambient lighting conditions, there were no failures, where the parameters acquired by a luminosity sensor were compared to a reference luxmeter, thus showing the necessary sensitivity to capture small variations. The dimerizing of the LEDs on the lampposts, thus generating varied lighting, was another important factor to obtain the results of this work. It is worth mentioning that an image can dimerize three lampposts, correctly distributing the light intensity to each of the three zones. Each of these zones correspond to an output of the Fuzzy algorithm modeled for the problem.

The people detection, including real environment and controlled environment, obtained satisfactory results, where objects (people) were detected in $83 \%$ of the images. It is worth mentioning that for application in a real environment (public ways), the camera must have some specific characteristics, such as CCD sensor (charge-coupled device), with a wide field of view and high sensitivity to light exposure (0.1 LUX), ensuring greater sensitivity in images to detect people in all areas of the image. Fig 6 shows two images from controlled environment used to validate the experiments.

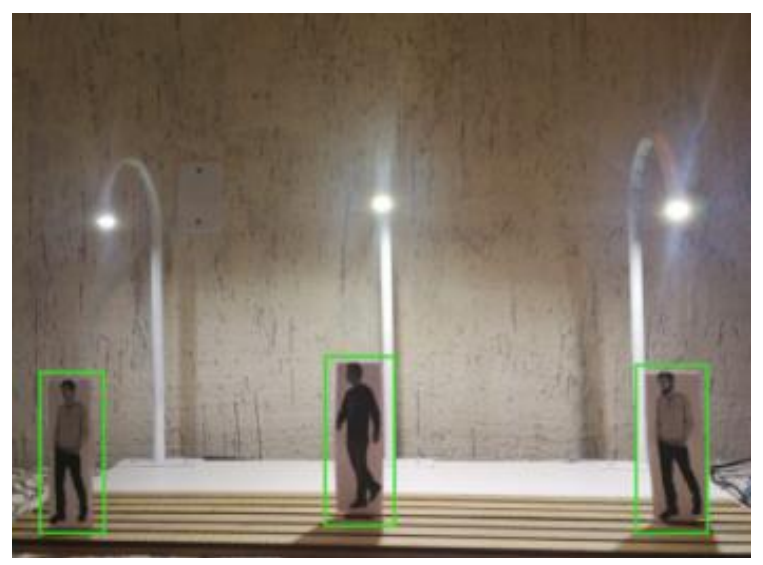




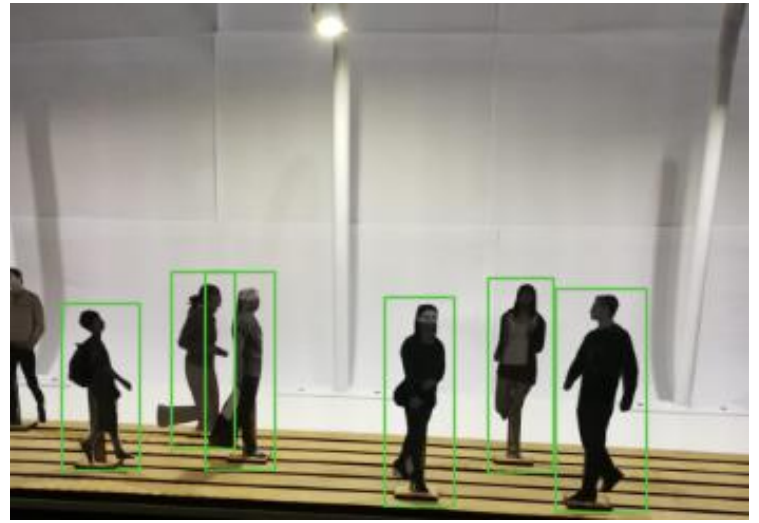

Figure 6. Example of image from controlled environment

Considering a street with three lampposts with of LED lamps $(280 \mathrm{~W})$, it is possible to calculate an approximate consumption of $10 \mathrm{kWh} /$ day, thus generating a monthly consumption $300 \mathrm{kWh} /$ month. Using the method proposed in this research and considering that the system is maintained within the minimal of $30 \%$ of power, without the pedestrian's presence, it is possible to calculate the reduction of energy consumption, $210 \mathrm{kWh} /$ month. These values are only considering LED lamps, when applied to sodium steam or mercury vapor lamps, this reduction in consumption is even greater, since these technologies need much more power to provide the same luminous flow. An example of a estimate scenario is described in table 3 .

Table 3. Estimate and calculation of consumption by time

\begin{tabular}{|c|c|c|c|c|}
\hline Timetables & People & $\begin{array}{c}\text { Period } \\
\text { On } \\
(\mathrm{min})\end{array}$ & $\begin{array}{c}\text { Output } \\
(\% \\
\text { power })\end{array}$ & $\begin{array}{c}\text { Consumption } \\
\text { Wh }\end{array}$ \\
\hline \multirow{5}{*}{$\begin{array}{c}\text { 7h pm to } 9 \mathrm{~h} \\
\mathrm{pm}\end{array}$} & 1 to 2 & 60 & 60 & 168 \\
\hline & 2 to 4 & 10 & 60 & 28 \\
\hline & 4 to 6 & 15 & 70 & 49 \\
\hline & 6 to 8 & 10 & 70 & 33 \\
\hline & 8 to 10 & 25 & 80 & 94 \\
\hline \multirow{5}{*}{$\begin{array}{c}9 \mathrm{~h} \mathrm{pm} \text { to } 0 \mathrm{~h} \\
\text { am }\end{array}$} & 1 to 2 & 80 & 60 & 224 \\
\hline & 2 to 4 & 30 & 70 & 98 \\
\hline & 4 to 6 & 20 & 80 & 75 \\
\hline & 6 to 8 & 20 & 90 & 84 \\
\hline & 8 to 10 & 30 & 100 & 140 \\
\hline \multirow{4}{*}{$\begin{array}{l}\text { Oh am to } \\
4 \mathrm{~h} 30 \mathrm{~m} \text { am }\end{array}$} & 0 & 60 & 30 & 84 \\
\hline & 0 & 60 & 30 & 84 \\
\hline & 0 & 60 & 30 & 84 \\
\hline & 0 & 90 & 30 & 126 \\
\hline \multirow{5}{*}{$\begin{array}{c}4 \mathrm{~h} 30 \mathrm{~m} \text { am to } \\
7 \mathrm{~h} \text { am }\end{array}$} & 1 to 2 & 60 & 80 & 224 \\
\hline & 2 to 4 & 40 & 70 & 130 \\
\hline & 4 to 6 & 20 & 70 & 65 \\
\hline & 6 to 8 & 20 & 60 & 56 \\
\hline & 8 to 10 & 10 & 60 & 28 \\
\hline $\begin{array}{c}\text { Consumption } \\
\text { per minute }\end{array}$ & 4.7 & & Total & 1874 \\
\hline
\end{tabular}

The consumption of one lamp per hour at full load is equivalent to $280 \mathrm{Wh}$. The consumption was calculated in $\mathrm{Wh}$ considering the time of each situation at timetables and period on. Considering a street with three lampposts using the proposed computer vision system we have a consumption of approximately $5.6 \mathrm{kWh} /$ day, in 30 days it is possible to estimate the consumption of $168 \mathrm{kWh} /$ month. This data may have a variation of $\pm 10 \%$ in consumption due to the routine of people who pass through the streets.

Comparing the proposed approach to employed nowadays is possible to simulate a reduction of $132 \mathrm{kWh} /$ month, or $44 \%$ in energy consumption. The results shown the importance of implementing these technologies on the streets, not only by reducing the costs of cities, but also preserving energy sources, thus making the whole system more effective and sustainable.

\section{CONCLUSION}

The proposed project presents a computer vision approach for public lighting management through image capture and analysis by calculating the flow of pedestrians on public ways, managing the power supplied for LED luminaires. The algorithm used was implemented in the Code::Blocks IDE, using $\mathrm{C} / \mathrm{C}++$ language and OpenCV image processing library. Experiments were performed in real and simulated environment, where it was possible to carry out several experiments, with all the conditions proposed in this research. The results were satisfactory because the system was stable in most of the conditions tested, having only a few false positives and false negatives with minimal lighting and less natural lighting.

The final hit rates computed were $83 \%$, considering that this rate can be increased with the use of specifics hardware, as describe in the results, thus obtaining a better quality in the acquisition of images under conditions of low incidence of light on the ways.

Considering the application in real environment of this project, it would be possible to obtain savings of approximately $44 \%$ in the consumption of energy spent on public lighting, thus generating real savings for the state, making it possible to distribute this value to other sectors, such as security or health.

It is noteworthy that this project can control a larger number of lamps than the three presented, requiring only the inclusion of new cameras, thus expanding the number of points served by the system. Other point is that the Raspberry Pi can be connected to the Internet, allowing in addition to remote monitoring, for example the use of the cameras for security purposes by public agencies, increasing the usefulness and reducing the cost of deployment due to its usefulness.

In future works, it is intended to use new techniques to recognize the people in the images, such as the use of convolutional neural networks, thus trying to increase the accuracy of the system and use of IP cameras with CCD sensor allowing to act in ambient with low luminous flow.

\section{ACKNOWLEDGMENTS}

The authors would like to thank UNINOVE by financial support for all authors.

\section{REFERENCES}

[1] V.B. Klein, J.L. Todesco, Um modelo conceitual para uso de Big Data e Open Data para Smart Cities, Int. J. Knowl. Eng. Manag. 6 (2017) 28-50. 
[2] C. Nwosu, A. Isiorhovoja, C. Ogbuka, B. Anyaka, Density based auto traffic light control system with GSM based remote override for enugu metropolis, J. Appl. Res. Technol. 18 (2020) 51-61.

[3] A.V. Rusu, D.D. Lucache, G. Livint, Assessment of Conflict Area Parameters Measurements by Using Photographic Methods, in: 2019 Int. Conf. Electromechanical Energy Syst., 2019: pp. 1-5.

[4] S.N. Jadhav, An energy efficient pedestrian aware Smart Street Lighting system, Int. J. Pervasive Comput. Commun. (2012).

[5] K.G. Di Santo, Active electricity demand management for consumers on smart grids, Universidade de São Paulo, 2018.

[6] R.C. Gonzalez, R.E. Woods, Digital Image Processing, 1st ed., Addison-Wesley, Massachusetts, 2002.

[7] Q. Zhu, M.-C. Yeh, K.-T. Cheng, S. Avidan, Fast human detection using a cascade of histograms of oriented gradients, in: 2006 IEEE Comput. Soc. Conf. Comput. Vis. Pattern Recognit., 2006: pp. 1491-1498.

[8] X. Wang, T.X. Han, S. Yan, An HOG-LBP human detector with partial occlusion handling, in: 2009 IEEE 12th Int. Conf. Comput. Vis., 2009: pp. 32-39.

[9] P. Mohandas, J.S.A. Dhanaraj, X.-Z. Gao, Artificial Neural Network based Smart and Energy Efficient Street Lighting System: A Case Study for Residential area in Hosur, Sustain. Cities Soc. 48 (2019) 101499.

[10] L.A. Zadeh, Fuzzy sets, Inf. Control. 8 (1965) 338-353.

[11] J.A. Galindo, M.V. Caya, Development of Street Lighting System with Object Detection, in: IEEE 10th Int. Conf. Humanoid, Nanotechnology, Inf. Technol. Commun. Control. Environ. Manag., 2018: pp. 1-5.

[12] S.P. Government, Executive Summary - Electricity Production and Consumption Data, São Paulo, Brasil, 2019. http://www.infraestruturameioambiente.sp.gov.br (accessed March 3, 2020).

[13] T.B. Marchesan, Integração de conversores estáticos aplicados a sistemas de iluminação pública, Universidade Federal de Santa Maria, 2007
[14] J.S. Brand, Análise da transferência de ondulação de baixa frequência em drivers de LEDs, Universidade Federal de Santa Maria, 2019.

[15] L. Schuch, M.A. Dalla Costa, C. Rech, L. Michels, G.H. Costa, A.S. dos Santos, Sistema Autônomo de Iluminação Pública de alta eficiência baseado em Energia Solar e LEDs, Eletrônica de Potência. 16 (2011) 17-27.

[16] J. Chen, R. Wu, Two-dimensional PCA-based human gait identification, Optoelectron. Imaging Multimed. Technol. $\quad$ II. $8558 \quad$ (2012) 85580D. https://doi.org/10.1117/12.999939.

[17] E.S. Poulsen, H.J. Andersen, O.B. Jensen, R. Gade, T. Thyrrestrup, T.B. Moeslund, Controlling urban lighting by human motion patterns results from a full scale experiment, in: Proc. 20th ACM Int. Conf. Multimed., 2012: pp. 339-348.

[18] S.P. Lau, G. V Merrett, A.S. Weddell, N.M. White, A traffic-aware street lighting scheme for Smart Cities using autonomous networked sensors, Comput. Electr. Eng. 45 (2015) 192-207.

[19] J. Jin, H. Huang, J. Sun, Y. Pang, Study on Fuzzy SelfAdaptive PID Control System of Biomass Boiler Drum Water, J. Sustain. Bioenergy Syst. 03 (2013) 93-98. https://doi.org/10.4236/jsbs.2013.31013.

[20] OpenCV - Open Source Computer Vision Library, OpenCV - Open Source Computer Vision Library, (2019). https://opencv.org/opencv-4-1/.

[21] N. Dalal, B. Triggs, Histograms of oriented gradients for human detection, in: 2005 IEEE Comput. Soc. Conf Comput. Vis. Pattern Recognit., 2005: pp. 886-893.

[22] A.C. Lorena, A.C. de Carvalho, Uma introdução às support vector machines, Rev. Informática Teórica e Apl. 14 (2007) 43-67.

[23] B.E. Boser, I.M. Guyon, V.N. Vapnik, A training algorithm for optimal margin classifiers. Proceedings of the fifth annual workshop on Computational learning theory; Pittsburgh, Pennsylvania, USA. 130401: ACM, (1992). 